Nota de investigación

\title{
Sostenibilidad en la cadena global de valor del arándano entre México y China
}

Arely Hernández Beltrán ${ }^{1 \S}$

Abel Pérez Zamorano ${ }^{1}$

Eduardo Campos Rojas ${ }^{2}$

Eusebio Dagoberto Torres Quintana ${ }^{1}$

${ }^{1}$ División de Ciencias Económico Administrativas. ${ }^{2}$ Fitotecnia-Universidad Autónoma Chapingo. Carretera México-Texcoco km 38.5, Texcoco, Estado de México, México. CP. 56230. (cemeesabelpz@gmail.com; educamro55@gmail.com; eusebio_torres@ hotmail.com).

${ }^{\S}$ Autora para correspondencia: arely.calmecac@gmail.com.

\section{Resumen}

La sostenibilidad tiene tres principios rectores: integridad ambiental, equidad social y prosperidad económica que se integran en el marco de triple bottom line. Ser sostenible implica integrar prácticas ambientales, sociales y económicas en los procesos de la cadena global de valor. La presente investigación tuvo como objetivo analizar las prácticas sostenibles y los impulsores de implementación en la industria del arándano azul. Se utilizó como estudio de caso la cadena global de valor del arándano entre México y China. Se recopiló información a través de entrevistas semiestructuradas, visitas a campo y fuentes de información secundaria. El análisis permitió identificar la existencia de tres tipos de impulsores para la implementación de prácticas sostenibles: el gobierno, los consumidores y las organizaciones no gubernamentales. Además, se identificaron el uso de genética y tecnología como práctica ambiental, el cumplimiento de leyes laborales como práctica social y el control del costo-calidad como práctica económica. Y se determinó que las certificaciones son usadas como herramienta de comprobación. Se concluyó que la cadena global de valor del arándano entre México y China, sigue presiones de mercado y prácticas de tipo económico, pero trabaja en iniciativas para mejorar sus prácticas ambientales y sociales.

Palabras clave: ambiental, cadena global de valor, económica, social.

Recibido: junio de 2021

Aceptado: julio de 2021 


\section{Cadenas globales de valor sostenibles}

Uno de los principales desafíos que enfrentan las cadenas globales de valor (CGV) es diseñar modelos de producción y de negocios que sean capaces no solo de satisfacer las demandas de los consumidores, sino también de ser sostenibles (Silva et al., 2019). La sostenibilidad tiene tres principios rectores: integridad ambiental, equidad social y prosperidad económica que se integran en el marco de triple bottom line (TBL). Al implementar el TBL en una CGV, las empresas pueden orientarse hacia una gestión sostenible, lo que incluye la preocupación por las ganancias, las personas y el planeta (Elkington, 2013).

Las empresas alcanzan la integridad ambiental al garantizar un daño ambiental mínimo en sus operaciones, equidad social mediante iniciativas de responsabilidad social y prosperidad económica a través de la creación de valor (Withiphakorn et al., 2019). El aspecto ambiental se aborda mediante el uso eficiente de recursos, el reciclaje y la reducción de desechos y emisiones. El aspecto social a través del cumplimiento de derechos humanos y leyes laborales, y el impacto en las comunidades locales. El aspecto económico se logra a través del desempeño económico de acuerdo con los indicadores operativos (Kovács y Illes, 2019).

El equilibrio entre el aspecto económico, ambiental y social se ha vuelto cada vez más importante para las empresas que enfrentan presiones sociales, regulatorias y de mercado (Shah y Siddiqui, 2019).

Las presiones, que a menudo son impulsadas por un gran número de partes interesadas (clientes, gobierno y grupos de presión), motivan a las empresas a adoptar prácticas sostenibles (D'Souza et al., 2020). En consecuencia, las prácticas sostenibles basadas en las dimensiones del TBL, muestran los esfuerzos de las empresas que integran la CGV, no solo en centrarse en la rentabilidad que pueden obtener, sino en el impacto ambiental, social y económico en su conjunto.

Aunque varios artículos se han acercado a la gestión sostenible de la CGV, hasta el momento no se han analizado las tres dimensiones en la industria del arándano azul, por lo que espera encontrar prácticas tanto económicas, como ambientales y sociales impulsadas por los consumidores. Esta investigación tuvo como objetivo analizar las prácticas sostenibles y los impulsores de implementación en la CGVAMCh, para saber quién promueve las prácticas a favor de la sostenibilidad y qué prácticas de sostenibilidad se llevan a cabo en la industria del arándano azul.

\section{El caso de la cadena global de valor del arándano entre México y China}

La investigación es de naturaleza exploratoria. Se llevó a cabo mediante un estudio de caso, en el que se estudiaron cinco frigoríficos y 12 predios autorizados en 2019 por el Servicio Nacional de Sanidad, Inocuidad y Calidad Agroalimentaria (SENASICA) y la administración general de supervisión de calidad, inspección y cuarentena (AQSIQ) para la exportación de arándano azul fresco mexicano a China (AGA, 2019). Se recopiló información a través de entrevistas semiestructuradas, visitas de campo y fuentes de información secundaria (Yin, 1998). Se analizó la información sobre prácticas sostenibles e impulsores de implementación en relación con la literatura de las dimensiones del TBL. 
El análisis se basa en la información obtenida de frigoríficos pertenecientes a la comercializadora Berries Paradise y predios pertenecientes a productores independientes que están conectados con la misma comercializadora; estos participantes fueron seleccionados porque ambos cumplen con los requisitos para participar en la CGVAMCh. La fuente de información más importante fueron 20 entrevistas semiestructuradas a trabajadores de la empresa y a expertos de la industria del arándano, a quienes se les presentó una lista de prácticas sostenibles, que se desarrolló a través del análisis de la literatura, vinculadas a cada dimensión de TBL (económica, social y ambiental) y que se complementaron con visitas de campo e información secundaria.

\section{Cadena global de valor}

Una cadena global de valor (CGV) vincula a empresas y consumidores mediante redes de producción y comercio, ofreciendo a empresas de países en desarrollo oportunidades para integrarse a la economía global (Gereffi, 2015).

La revisión de literatura muestra que el análisis de una $\mathrm{CGV}$ es particularmente útil para comprender la dinámica global de una industria, en cuatro dimensiones: 1) estructura de entradasalida de un producto vinculado a una secuencia de actividades de agregación de valor; 2) consideración geográfica de la dispersión espacial de una red de producción o distribución compuesta por empresas de diferentes tamaños y tipos; 3) estructura de gobernanza que muestra la relaciones de autoridad y poder y el tipo de escalamiento que se permite; y 4) contexto institucional (Gereffi y Fernandez-Stark, 2011).

Sin embargo, en años recientes no solo se estudia la CGV a través del análisis del papel que juegan las empresas que la integran, donde se ubican, que actividades realizan, cómo y quién organiza la cadena, cómo se mejoran las actividades y que regulación rige la cadena, sino también a través de sus prácticas sostenibles. La CGV es sostenible si lleva a cabo prácticas ambientales, sociales y económicas incluidas en las interacciones entre sus eslabones.

\section{Prácticas sostenibles}

La cadena global de valor del arándano entre México y China se conforma de tres eslabones principales: producción, comercialización y distribución. Las prácticas sostenibles en el contexto de dicha CGV se relacionan con el marco de TBL, que se basa en la integración de prácticas sociales, ambientales y económicas, que ayudan a la creación de valor. Las prácticas sostenibles, identificadas en la CGVAMCh, que impactan positivamente en la imagen de las empresas que la integran (productores, comercializadoras y distribuidores) y de la industria del arándano azul en general, se enfocan, por tanto, en el aspecto ambiental, social y económico (Figura 1).

En el aspecto ambiental sobresalen las prácticas de producción más limpias. Algunas de las acciones ambientales encontradas corresponden al uso de nuevas variedades 'Sophia', la eliminación planificada de envases de agroquímicos, la reducción en el uso de insecticidas para proteger a los polinizadores e insectos benéficos, el diseño del proceso de producción incorporando el principio de eficiencia en el uso del agua (sistema de riego por goteo, sustrato y túneles), la adquisición y uso de moléculas permitidas en el mercado de exportación que cumplen con el 
registro sanitario consolidado (RSCO) y el empleo óptimo de las mismas, y un buen manejo integrado fitosanitario. Se trabaja en prácticas para la medición de huella hídrica, el uso de tecnologías de precisión y el uso de envases biodegradables.

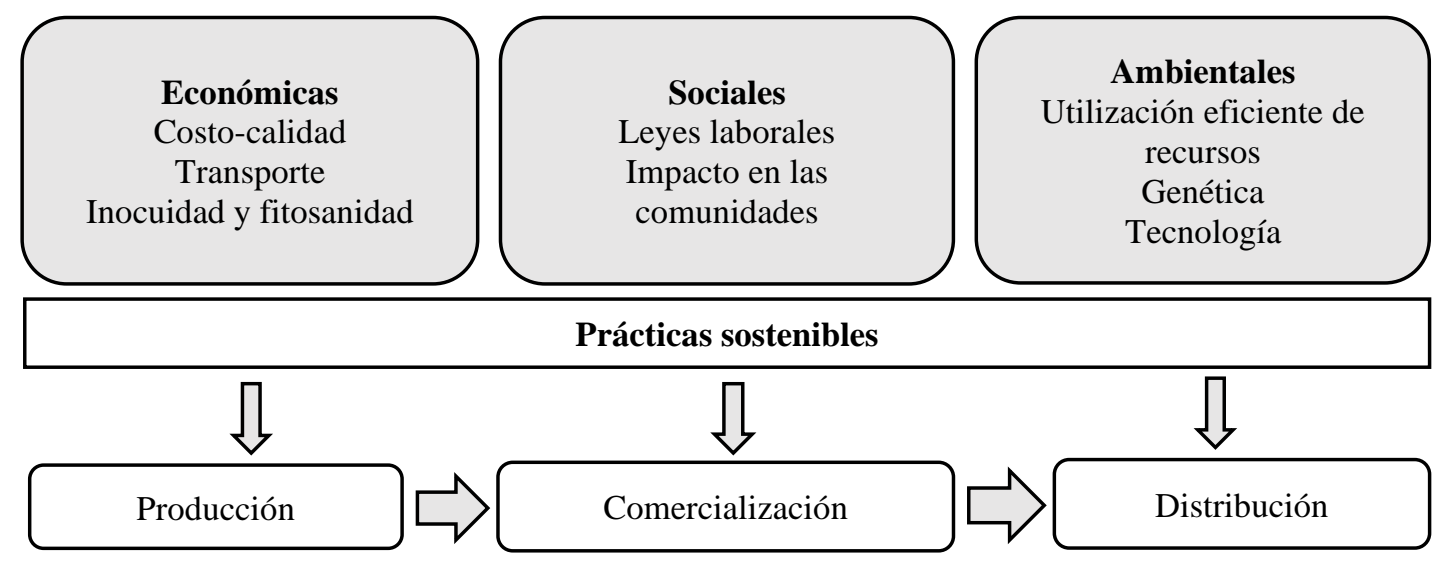

Figura 1. Prácticas económicas, sociales y ambientales en la CGVAMCh.

Respecto al aspecto social, las empresas buscan relacionarse con las comunidades circundantes al desarrollar proyectos sociales (apoyo a iniciativas de la comunidad para el mejoramiento de infraestructura, actividades deportivas, etc.), siguen leyes nacionales e internacionales contra la explotación laboral y trabajo infantil, generan empleo en la comunidad local (la cosecha, por ejemplo, es una actividad intensiva en mano de obra que requiere entre 8 a 10 jornaleros por ha), ingreso digno (de tres a seis salarios mínimos en cosecha), buenas condiciones laborales (lugares de trabajo seguros y limpios), y donan fruta a organizaciones benéficas.

En el aspecto económico, las empresas que participan en la CGVAMCh mantienen sus negocios rentables a través de procesos que les permiten responder rápidamente a las necesidades de sus clientes y a los cambios del mercado, mientras controlan los costos y la calidad del producto y con ello obtener crecimiento en sus ventas, utilidades y cuotas de mercado. Algunas actividades que permiten dicho control son: la optimización de cargas y rutas de transporte al acortar tiempos o costos, el uso de temperaturas adecuadas en el almacenamiento y transporte, la inspección de material de envase, empaque y embalaje para asegurar que cumplan con todos los requisitos de calidad y seguridad y la verificación del producto en el cumplimiento de requisitos de calidad, inocuidad y fitosanidad para evitar rechazos y pérdidas.

Las principales barreras de implementación de prácticas sostenibles son: el costo de implementación, los recursos de los que se dispone y el conocimiento con el que se cuente; sin embargo, la adopción de dichas prácticas está inspirada por los valores y creencias de cada actor que conforma la CGVAMCh. Las prácticas sostenibles usadas en la industria son promovidas por la Asociación Nacional de Exportadores de Berries (Aneberries); a través, de la impartición de cursos sobre temas de sostenibilidad ambiental y social, reforzadas por la alianza hortofrutícola internacional para el fomento de la responsabilidad social (Ahifores) y apoyadas directamente por la comercializadora, que se ha vuelto más activa en la promoción de prácticas sostenibles para luego vincularlas a su marca y con ello mejorar su competitividad y valor comercial. 


\section{Impulsores de implementación}

Las prácticas económicas, sociales y ambientales implementadas en la cadena global de valor de arándano entre México y China son impulsadas por diversos actores que ejercen diferentes presiones (Figura 2).

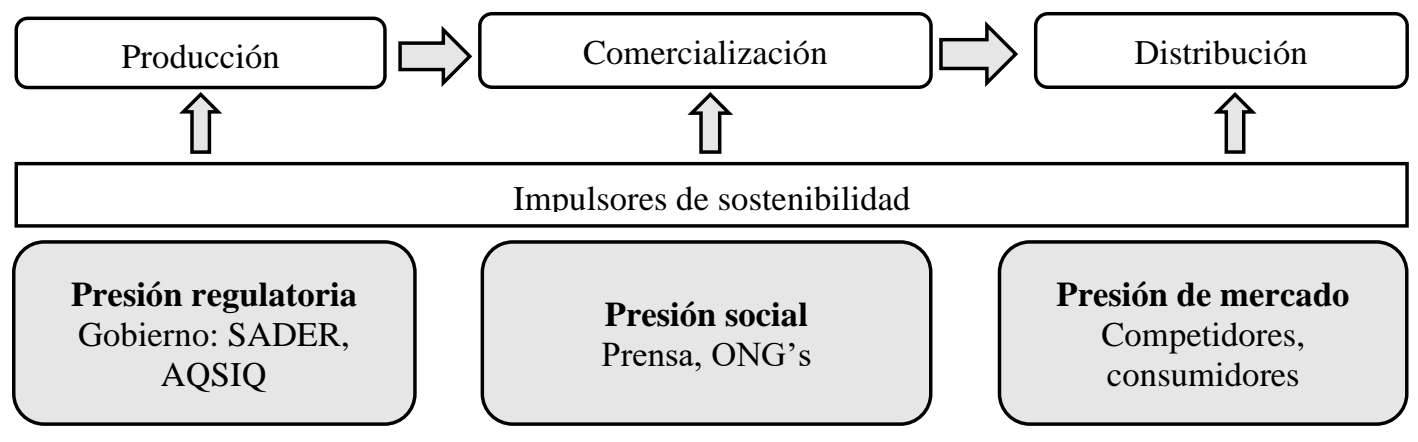

Figura 2. Presiones regulatorias, de mercado y sociales en la CGVAMCh.

Agencias gubernamentales como la Secretaría de Agricultura y Desarrollo Rural (SADER) en México y administración general de supervisión de calidad, inspección y cuarentena (AQSIQ) en China, ejercen presión regulatoria sobre productores y la comercializadora para que cumplan con los requisitos establecidos. La presión regulatoria en la cadena global de valor está relacionada con la verificación de las instalaciones de cosecha, de empaque, de almacenamiento y de transporte, para comprobar que cumplen con los lineamientos establecidos en el protocolo de requerimientos fitosanitarios para la exportación de frutos frescos de arándanos de México a China.

La presión de mercado ejercida por los consumidores sobre temas de sostenibilidad aumenta el reconocimiento y reputación de una marca cuando las empresas que integran la CGVAMCh cumplen con sus expectativas, y la presión ejercida por los competidores les brinda oportunidades de aprendizaje sobre nuevas prácticas de sostenibilidad. Por tanto, la presión de mercado impulsa a las empresas que participan en la CGVAMCh a adoptar prácticas de sostenibilidad sociales y ambientales que les permita obtener una ventaja competitiva sobre sus competidores y mejorar su relación con los consumidores.

La presión social, ejercida principalmente por las ONG's y medios de comunicación, enfoca su atención en cómo las empresas que participan en la CGVAMCh manejan los problemas ambientales (manejo del recurso hídrico, residuos, insumos, pesticidas, etc.) y los derechos de los trabajadores (vivienda, salud, capacitación, salario, etc.) y aumentando la conciencia pública.

\section{Certificaciones}

Si bien, las empresas participantes de la CGVAMCh pueden emplear prácticas económicas, sociales y ambientales, los esquemas de certificación tienen un impacto creciente en su capacidad para generarlos y demostrarlos. Las empresas que conforman la CGVAMCh se rigen y se evalúan por certificaciones que controlan las condiciones sanitarias y fitosanitarias, la trazabilidad, el uso de plaguicidas, etc. Por ejemplo, global good agricultural practice (Global GAP) incluye criterios de cumplimiento para todas las etapas de producción, desde las actividades previas a la cosecha, 
como el manejo del suelo y el uso de fertilizantes, hasta las actividades posteriores a la cosecha, como el embalaje y el almacenamiento, incluyendo temas de inocuidad, ambientales y bienestar de los trabajadores.

Las empresas que conforman la CGVAMCh evalúan el costo-beneficio que ofrecen las certificaciones, si encuentran un aumento en la demanda, una reducción de fallas mediante el reforzamiento de la calidad e inocuidad y una mejora de los procesos de producción y comercialización, la mantendrán; y aunque las certificaciones pueden dar una indicación del estado de la sostenibilidad, son solo una guía, las prácticas implementadas hasta ahora indican que el enfoque de la CGVAMCh hacia la sostenibilidad siguen presiones del mercado y prácticas de tipo económico, que les permiten acceder al mercado de China.

\section{Conclusiones}

La principal razón por la cual las empresas que conforman la CGVAMCh se interesan en el tema de sostenibilidad es para garantizar el cumplimiento de los requisitos de sus clientes. Sin embargo, ello les permite llevar a cabo acciones que resultan en mejores impactos sociales, económicos y ambientales. Con respecto al aspecto económico, las empresas promueven su propio crecimiento e ingresos, en el aspecto social ayudan a mejorar el nivel de vida de la comunidad donde se establecen y el de sus empleados, en el aspecto ambiental disminuyen el consumo de recursos y la contaminación. Las certificaciones son importantes para la producción y el comercio de arándanos, ya que proporcionan garantía del cumplimiento de normas laborales, ambientales o comerciales, que les permite mantener su posición comercial. Además, se trabaja con otras estrategias que den la oportunidad de diferenciarse y hacer más con menos.

\section{Literatura citada}

AGA. 2019. Febrero, 25. Administración general de aduanas de la república popular china lista de huertos y plantas de empaque registrada de moras, frambuesas y arándanos exportados de México a China. http://www.customs.gov.cn/customs/jyjy/dzwjyjy/qymd/zwjcp/ 2163997/index.html.

D-Souza, C.; McCormack, S.; Taghian, M.; Chu, M. T.; Mort, G. S. and Ahmed, T. 2020. An empirical examination of sustainability for multinational firms in china: implications for cleaner production. J. Cleaner Production. 242:1-12. Doi: 10.1016/j.jclepro.2019.118446.

Elkington, J. 2013. Enter the triple bottom line. In: the triple bottom line: does it all add up? henriques, A. and Richardson, J. (Ed.). Routledge. 23-38 p. Doi: 10.4324/9781849773348.

Gereffi, G. 2015. América Latina en las cadenas globales de valor y el papel de china. Boletín Informativo Techint. 350:27-40.

Gereffi, G. and Fernandez-Stark, K. 2011. Global value chains analysis: a primer $1^{\mathrm{a}}$ (Ed). Center on globalization governance and competitiveness (CGGC), duke University, North Carolina, USA. 1-39 pp.

Kovács, G. and Illés, B. 2019. Development of an optimization method and software for optimizing global supply chains for increased efficiency, competitiveness, and sustainability. Sustainability. 11(6):1-28. Doi: 10.3390/su11061610.

Shah, A. and Siddiqui, D. A. 2019. Customer's driven green supply management and organization performance. Social Sci. Humanit. J. 3(4):1054-1067. Doi: 10.18034/gdeb.v8i2.99. 
Silva, W. H.; Guarnieri, P.; Carvalho, J. M.; Farias, J. S. and Reis, S. A. D. 2019. Sustainable supply chain management: analyzing the past to determinate a research agenda. Logistics, 3(2):1-15. Doi: 10.3390/logistics3020014.

Withisuphakorn, P.; Batra, I.; Parameswar, N. and Dhir, S. 2019. Sustainable development in practice: case study of l'oréal. J. Bus. Retail. Manag. Res. 13(spe):35-47. Doi: 10.24052/jbrmr/v13issp/art-4.

Yin, R. K. 1998. The abridged version of case study research: design and method. In: handbook of applied social research methods. Bickman, L. and Rog, D. J. (Ed.). Sage Publications. USA. 229-259 pp. 